\title{
Implementación de la cultura empresarial para el mejoramiento del desempeño laboral.
}

\author{
Implementation of the corporate culture to improve work \\ performance.
}

\author{
Ketty Marilú Moscoso Paucarchuco ${ }^{1 *}$, Ing. María América Villafuerte Quiroz ${ }^{2}$ \\ 1.* Universidad Nacional Autónoma de Huanta, Perú. Email: kmoscoso@unah.edu.pe \\ ORCID: https://orcid.org/0000-0003-2097-8658
}

2. Ingeniera en Contabilidad Superior Auditoria y Finanzas CPA, Investigadora Independiente, Ecuador. Email: mery_scarleth@hotmail.com ORCID: https://orcid.org/0000-0003-3202-6080

Destinatario:kmoscoso@unah.edu.pe

Recibido: 30/febrero/2021 Aceptado: 30/marzo/2021 Publicado: 30/abril/2021

Como citar: Moscoso Paucarchuco, K. M., \& Villafuerte Quiroz, M. A. (2021). Implementación de la cultura empresarial para el mejoramiento del desempeño laboral. Revista E-IDEA Journal of Business Sciences, 3(11), 25-35. https://doi.org/10.53734/eidea.vol3.id90

\begin{abstract}
Resumen: Actualmente la cultura empresarial y el desempeño laboral comienzan a formar parte del motor de una organización. La importancia de las mismas empiezan a ser reconocidas por altos mandos como una forma de lograr mayor productividad y competitividad, las organizaciones desempeñan un papel importante para la sociedad, ya que genera bienes y servicios para satisfacer las necesidades de las personas. El objetivo de este artículo, es estudiar la importancia de la implementación de la cultura empresarial para el mejoramiento del desempeño laboral de los trabajadores. La metodología empleada en esta investigación se basa en un diseño bibliográfico de tipo documental. Como Conclusión se menciona que para que el desempeño laboral de los empleados sea productivo y sostenible en el tiempo, es necesario que exista un ambiente motivador en la empresa, donde al dar mayor responsabilidad se pueda recompensar los logros obtenidos. Con esto se ofrece, satisfacción laboral para que los trabajadores eleven su desempeño. Es así como, la productividad de una organización o empresa depende de un buen desempeño de los trabajadores, por lo que, a mayor rendimiento y mayor bienestar para los mismos, mejor será su calidad.
\end{abstract}

Palabras Clave: Cultura Empresarial, Desempeño Laboral, productividad, competitividad.

\begin{abstract}
Currently, business culture and job performance are becoming part of the engine of an organization. Their importance is beginning to be recognized by senior managers as a way to achieve greater productivity and competitiveness. Organizations play an important role for society, since they generate goods and services to meet people's needs. The objective of this article is to study the importance of the implementation of the corporate culture for the improvement of the work performance of the workers. The methodology used in this research is based on a documentary-type bibliographic design. As a Conclusion it is mentioned that for the work performance of employees to be productive and sustainable over time, it is necessary that there be a motivating environment in the company, where by giving greater responsibility the achievements obtained can be rewarded. With this, job satisfaction is offered for workers to increase their performance. Thus, the productivity of an organization or company depends on a good performance of the workers, so that the higher the performance and greater well- being for them, the better their quality will be.
\end{abstract}

Keywords: Business Culture, Work Performance, productivity, competitiveness. 


\section{INTRODUCCIÓN}

$\mathrm{E}$ n la actualidad las organizaciones han venido transformando sus procesos y estructuras organizacionales para poder adaptarlas a las características de los constantes cambios y así mantener un nivel de exigencia mayor en referencia a su desempeño, y para eso es necesario conocer claramente la cultura de la organización ya que constituye una forma de estimar las posibilidades de éxito en la misma.

Se sabe, que una organización es un grupo de personas con funciones específicas, que actúan en conjunto para lograr alcanzar un propósito determinado que va de la mano con la alta gerencia. Toda organización tiene un objetivo o una meta trazada una estructura y una población de personas que se involucran en los procesos, y precisamente es relevante estudiar el desempeño laboral de estas personas.

Ante el contexto anterior, las empresas e instituciones están tomando en cuenta el tema de la cultura organizacional como variable fundamental para mejorar el desempeño de sus colaboradores, por medio de herramientas y decisiones que guíen la actividad organizacional y las influencias externas que finalmente determinan el desempeño y la productividad.

En efecto, es importante que las empresas conozcan el comportamiento de ciertos indicadores que les den directrices hacia la mejor toma de decisión, dos de estos indicadores son: La Cultura Empresarial y El Desempeño Laboral.

En este artículo su busca estudiar la implementación de la cultura empresarial para el mejoramiento del desempeño laboral, para lo cual se llevó a cabo la investigación donde se utilizó la revisión documental y así analizar las implicaciones de la cultura empresarial, estudiar el desempeño laboral de los trabajadores para luego resaltar la importancia de implementar la cultura organizacional como herramienta para el mejoramiento del desempeño laboral de los trabajadores.

\section{METODOS}

La metodología empleada en esta investigación se basa en un diseño bibliográfico de tipo documental a través de la revisión de artículos científicos indexados en bases de datos con verificable rigor académico se concreta exclusivamente en la recopilación de información de diversas fuentes, con el objeto de organizarla describirla e interpretarla de acuerdo con ciertos procedimientos que garanticen confiabilidad y objetividad en la presentación de los resultados (Palella Stracuzzi y Martins Pestana, 2010). 


\section{RESULTADOS Y DISCUSIÓN}

\section{Cultura empresarial.}

La cultura empresarial se puede definir en atención a Granados (2017) como el conjunto de normas, valores y formas de pensar que caracterizan el comportamiento del personal en todos los niveles jerárquicos de la empresa, el estilo de la dirección, la forma de asignar los recursos y de la forma de organizar internamente, así como la imagen de la empresa. Es así como la cultura empresarial se presenta por medio de símbolos, rituales, mitos y otras expresiones donde los valores están ordenados por una preferencia jerárquica.

En este orden de ideas, en la bibliografía se aprecian definiciones similares; tal es el caso de la Cultura Corporativa Espinoza y Gaspar (2018) que es el conjunto de creencias que se convierten en valores, en pautas y modos de comportamiento, inventadas, descubiertas o desarrolladas por un grupo de trabajadores que al ir aprendiendo a enfrentarse con sus problemas de adaptación externa o integración interna se consideran válidas, y que en consecuencia merecen ser enseñadas a los nuevos miembros como el modo correcto de percibir, pensar y sentir esos problemas.

Mientras que, la Cultura organizacional, es un sistema de significados compartido entre los miembros de una empresa y que la distingue o diferencia de las otras. Al examinar con mayor detalle este sistema de significado compartido, se identifica un grupo de características clave que la organización valora (Mendez, 2019). Tomando en consideración los conceptos anteriores, a efectos de este artículo, la cultura empresarial es sinónimo de cultura organizacional.

En opinión de, Calero (2018) la cultura organizacional tiene básicamente dos funciones: "una interna, referida a la integración, y una externa, que es la adaptación". Con lo cual se puede decir, que el empleado una vez que está dentro de la organización se integra a la nueva cultura, pero también debe adaptarse a ese medio que la considera externa, de acuerdo a los factores que la determinan, por ejemplo: la experiencia en otras empresas, el grado de preparación profesional que haya tenido y finalmente sus paradigmas que motivan a un cambio de mentalidad.

\section{Tipos de Cultura Organizacional}

De acuerdo a lo propuesto por Flebes y Oreja (2018) la cultura organizacional se divide en cuatro tipos:

1. Cultura de participación: donde por medio de la autonomía, creatividad e iniciativa individual, se busca desarrollar al personal, fomentando la motivación para su participación en la creación de objetivos y toma de decisiones

2. Cultura de compromiso: Se relaciona con la existencia de una preocupación por el bienestar de los empleados y su motivación, y la importancia otorgada a la identificación del trabajador con los valores y creencias de la empresa; 


\section{E-IDEA}

J OURNAL OF B USINESS SCIENCES

3. Cultura de adaptación: busca asignar objetivos claros y concretos a los empleados para que no existan desviación con respecto a la planificación del cambio estratégico y se evite la resistencia al cambio. Este tipo de cultura, destaca lo importante que es la capacidad de adaptación de la empresa a los cambios y fomenta la flexibilidad, la comunicación y la cooperación.

4. Cultura de formación: es un tipo de cultura en la que se hace especial énfasis en la formación específica del trabajador para su puesto de trabajo, orientándolo criterios de eficiencia en el trabajo. Con esto se busca controlar los procesos internos de trabajo.

De manera similar, Robbins, S. y Coulter (2014) exponen la existencia de "una cultura dominante y las subculturas, entendiéndose por dominante la que sintetiza los valores principales y esenciales que comparten los integrantes de la organización y que marca la personalidad distintiva". Mientras que, cuando se refiere a "las subculturas tienden a desarrollarse principalmente en las organizaciones grandes para manifestar conflictos o experiencias en quienes se pertenecen al mismo departamento o lugar". En otras palabras, la cultura fuerte es aquella en que la mayoría de empleados comparten el mismo criterio acerca de la misión y los valores de la organización, se adaptan a ella de forma inmediata, presenta compromiso de acción es basado en valores; mientras que, si esto no es así, la cultura se considera débil.

\section{Elementos de la cultura organizacional}

El tema de los elementos de la cultura organizacional abarca el entorno externo de la empresa, es decir, sobre lo que no tiene un control directo porque depende de otras personas como son los clientes, proveedores, competidores, autoridades que crean, modifican o derogan leyes, entre otros; y los que maneja de manera interna la empresa, como factores internos.

Existen diferentes elementos que constituyen la cultura organizacional Raffino (2020) y se utilizan de manera conjunta. Estos elementos son:

- Identidad de la organización: La misión, la visión y los valores de la empresa, definen la cultura empresarial.

- Sistemas de control: que permitan a la cultura organizacional tener sistemas que vigilen aquello que está sucediendo de manera interna de la empresa con el capital humano (empleados y directivos).

- Estructuras de poder: es necesario que la cultura empresarial establezca quiénes son las personas encargadas del proceso de toma de decisión, distribuyendo el poder y autoridad.

- Símbolos: La cultura organizacional está formada por todos los diseños, bien sea auditivos o visuales que forman parte de la identidad de la empresa.

- Rituales y rutinas: esto contempla o constituye todas las reuniones empresariales o de negocios, informes de desempeño que ocurran dentro de la organización. 
- Historias, mitos y anécdotas: Las historias cuentan el inicio y evolución de las organizaciones, su impacto actual en el mercado. Las narraciones de las anécdotas por parte de los trabajadores con más tiempo a los nuevos empleados, enriquecen el conocimiento y el sentido de pertenencia.

Dentro de las ventajas que se tiene con la cultura empresarial se encuentran las definidas en la Figura1

Figura 1.

Ventajas de la Cultura Organizacional:

\begin{tabular}{|c|c|}
\hline $\begin{array}{l}\text { 1. Direcciona comportamientos en la } \\
\text { interacción social }\end{array}$ & $\begin{array}{l}\text { Cada organización construye parámetros que orientan el } \\
\text { comportamiento de las personas, para que actúen de manera } \\
\text { similar en aspectos formales e informales de la dinámica, } \\
\text { validando el concepto de conciencia colectiva, Los fundadores } \\
\text { y/o lideres, señalan parámetros como la ideologia, los } \\
\text { valores, la responsabilidad social y la ética; la organización, } \\
\text { politicas, normas, ritos, símbolos, himno, bandera, logotipo), } \\
\text { ylas personas historias, creencias mitos, lenguaje, y clima } \\
\text { organizacional. }\end{array}$ \\
\hline 2. Propicia identidad y cohesión & $\begin{array}{l}\text { La cultura propicia la identidad de las personas, condición } \\
\text { necesaria para la organización. Se logra en la medida que } \\
\text { se cohesionan socialmente, compartiendo, aceptando y } \\
\text { proyectando en su desempeño lineamientos propios que se } \\
\text { reflejan en el nivel de compromiso, la responsabilidad social, } \\
\text { los valores, la ética y las normas con los que actúan. asi como } \\
\text { en el sentido de pertenencia de los colaboradores, quienes } \\
\text { tienen conciencia de su responsabilidad individual en el rol } \\
\text { que desempeñan. }\end{array}$ \\
\hline 3. Factor de Diferenciación & $\begin{array}{l}\text { Las organizaciones son referentes dificilmente imitables } \\
\text { por sudinámicas, ya que estas no son sencillas de asimilar y } \\
\text { adaptar en otras orgnizaciónes; por tal razón, no se encuentran } \\
\text { organizaciones similares en su cultura inclusive de un grupo } \\
\text { empresarial con el mismo fundador. }\end{array}$ \\
\hline $\begin{array}{l}\text { 4. Normas de acción } \\
\text { (las determina Estructura) }\end{array}$ & $\begin{array}{l}\text { A nivel formal, las normas se establecen desde la estructura, } \\
\text { que en sus procesos, procedimientos, manuales de funciones, } \\
\text { no dan alternativa de actuar fuera de lo estipulado, lo que se } \\
\text { afianza con las normas aplicadas en la acción. }\end{array}$ \\
\hline
\end{tabular}

Fuente: (Mendez, 2019)

\section{Desempeño Laboral}

Hablar del desempeño del personal involucra evaluar lo que de verdad realizó una persona en cierto espacio de tiempo, $y$, a partir de aquello, concretar decisiones respecto a cada empleado; como lo afirma Loufatt (2012), que históricamente fue vista como elemento clave relacionado al castigo o la sanción. Cuando en realidad se trata de corregir, mejorar, solucionar las debilidades del personal y maximizar las potencialidades del trabajador. 
Es necesario tener claro que el concepto de desempeño laboral tiene variedad de definiciones, debido al aporte que brinda cada autor u organización, así por ejemplo:

En opinión de Robbins (2004) se refiere al desempeño laboral como un conjunto de habilidades físicas y psicológicas que se necesitan para desarrollarse en un puesto de trabajo. Detalla que esta se ve favorecida cuando las habilidades y aptitudes de la persona corresponden idóneamente a su puesto; sin embargo, enfocarse únicamente en el puesto y no en las habilidades es llevarlos al fracaso, ya que el desempeño debe ir enfocado en la búsqueda de la satisfacción laboral de ellos mismos.

Mientras que Toro (2002) concibe el desempeño como la acción o acciones que un sujeto ejecuta con el ánimo de obtener un resultado. En el trabajo puede incluir conductas orientadas al cumplimiento de las responsabilidades del cargo y al desarrollo de procesos inherentes a este (desempeño intrarrol), la realización de actividades extras que agregan valor (desempeño extrarrol) o actuaciones coherentes con las normas y principios definidos por la organización (cumplimiento de normas) Toro F (2010)

La evaluación de desempeño facilita el análisis periódico de la actuación de cada integrante de la organización de modo de obtener la información de mayor calidad para lograr la utilización más eficaz de los recursos humanos con que cuenta, de manera tal que cada uno de ellos contribuya con el máximo de su capacidad al logro de los objetivos, tanto organizacionales como individuales. Para estudiar la evaluación del desempeño es importante tomar en consideración como elementos claves, el control y la supervisión, ya que ambos periten corregir y mejorar los planes iniciales, medir la eficacia de las actividades y fundamentar la toma de decisiones y en caso de ser necesario plantear opciones (Sira, 2020)

Si la empresa quiere permanecer siendo competitiva, debe estar en la disposición de cambiar y mejorar las relaciones con las personas y sus talentos a fin de poder evolucionar a una empresa que interaccione más con sus clientes y trabajadores. Toda empresa debe contar con un adecuado sistema de evaluación del desempeño. Esta herramienta permite describir y visualizar las metas, para lograr altos niveles de competitividad en una organización (De LaTorre y Afan, 2017)

De todo lo expuesto anteriormente, se puede inferir que el desempeño de las organizaciones depende en gran medida del entorno en el que se desenvuelva. La cultura empresarial es un determinante del desempeño ya que impacta no solo los resultados directos y la eficiencia de una organización, sino también la salud, el entusiasmo, el sentido de pertenencia y el compromiso de su personal

\section{Implementación de la cultura para mejoramiento del desempeño laboral}

Las empresas deben tener conocimiento de cómo es su cultura empresarial ya que esta expresa claramente las actitudes, conductas y valores que tiene yasí analizar cómo relacionarla con el desempeño laboral de sus trabajadores, ya que en la medida que una

Moscoso Paucarchuco, K. M., \& Villafuerte Quiroz, M. A. (2021). Implementación de la cultura empresarial para el mejoramiento del desempeño laboral. E-IDEA Journal of Business Sciences, 3(11), 
buena o mala cultura organizacional se manifieste, será igualmente decisiva en el desempeño laboral personal (De LaTorre y Afan, 2017).

Ahora bien, desde un punto de vista interno, las empresas se benefician al tener empleados con percepciones positivas de la empresa ya que identificándose con los valores de la compañía, mayor será el sentido de pertenencia y mejor percepción del desempeño general de la empresa, generando mayor satisfacción laboral en los empleados Diehl, Schaefer, y Terlutter (2019), que se traduce en mejoras del desempeño individual de cada individuo dentro de la empresa (Lee, Lee, y Youn, 2018).

En este orden de ideas, como ya se ha mencionado por medio de algunos autores en este documento, el bienestar en los lugares de trabajo de los empleados es fundamental para que su desempeño se optimice, como comentan Su y Swanson (2019) al sugerir que la cultura organizacional provee un camino para entender mejor el bienestar laboral y el retorno de beneficios que genera. En otras palabras, existe una relación positiva entre la implementación de la cultura organizacional y el mejoramiento del desempeño laboral de los empleados.

La cultura organizacional caracteriza a la empresa y por lo tanto marca la diferencia por eso debe estar bien definida Para que la cultura de la empresa pueda ponerse de manifiesto, es necesario que tanto la alta gerencia como todos los niveles funcionales dela organización tengan conocimiento sobre la misión y la visión. La misión y la visión reflejan el porqué de la existencia de la empresa, los objetivos, la ideología. Todos los integrantes de la organización deben conocer y tener claro la misión y visión ya que estas darán directrices y así todos dirigirán esfuerzos a los mismos objetivos afianzando la cultura organizacional.

De esta manera según Granados (2017) se puede decir que una de las formas en que las empresas pueden lograr transmitir la cultura organizacional a sus empleados es:

- con el compromiso de la gerencia de ejecutar actividades para que los empleados perciban la importancia de tener una identidad como organización. La forma de transmitir, a los empleados la ideología de la empresa es teniendo una buena comunicación, así como tener claros los objetivos de todos los aspectos que hacen en la empresa.

- Garantizando el buen flujo de la información, confianza de los supervisores y empleados en la comunicación, participación en la toma de decisiones, canales abiertos a todo tipo de comunicación, además de la correcta retroalimentación.

- Fomentando un estilo de liderazgo centrado en el empleado, amplios procesos de interacción. Se genera en la organización conciencia de que es un sistema, donde todas sus partes se interrelacionan.

- Capacitar de forma permanente a todos los integrantes de la organización, para que estén en condiciones de tomar decisiones y así mantener la calidad de la empresa.

Implementar una cultura empresarial fuerte tiene relación con un desempeño alto de los colaboradores en las diferentes organizaciones por tres simples razones Jiménez (2016). 
La primera razón es porque una cultura fuerte se ajusta entre la estrategia de la organización y la cultura que tiene la misma. Con esto se logra cumplir con éxito las estrategias y objetivos que se plantea la empresa. La segunda razón en la cual la cultura organizacional fuerte influye en el desempeño laboral, es que generalmente los trabajadores son participes de los objetivos que fijan la organización y se sienten identificados con ella. Y por último la tercera razón, es que impacta al personal de una organización haciendo que sienta el compromiso y la motivación para poder trabajar de una manera eficaz y eficiente.

Finalmente, al contar con una Cultura Organizacional fortalecida, conocida y bien definida, se produce un incremento en el desempeño laboral y por ende en la productividad.

\section{CONCLUSIONES}

La cultura de una empresa está constituida por las condiciones, la tradición y los valores que dan lineamientos para las acciones de los trabajadores. Esta cultura puede tener características tales como confianza, trabajo en equipo y participación. Es de hacer notar que las culturas donde son más humanizadas las condiciones de trabajo, aumentan la productividad, los ingresos por ventas y la autoestima de los empleados.

Conocer la cultura empresarial en una organización es de gran importancia, ya que permite entender las conductas y valores que tiene cada uno de los empleados, lo cual constituye y ayuda al desempeño laboral.

A cultura organizacional tiene dos funciones, la primera es la integración entre sus miembros y la segunda es que permite a la organización adaptarse al entorno,

La evaluación de desempeño laboral es uno de los ejes principales en una organización ya que además de orientar a la empresa, permite medir el rendimiento de los trabajadores y determinar que les falta a los mismos para mejorar su rendimiento.

Se recomienda fomentar la cultura organizacional, estableciendo políticas enfocadas en la mejora del clima laboral, así como filosofías de trabajo que como se analizó se relaciona directamente con el desempeño laboral de los trabajadores con lo cual se obtienen mejoras en el rendimiento. 


\section{REFERENCIAS BIBLIOGRÁFICAS}

Calero, F. (2018). La cultura organizacional y el desempeño del personal en las empresas procesadoras de atún, de la provincia de Manabí - Ecuador. Lima, Perú: Trabajo especial de grado de la uUniversidad Nacional Mayor de San Marcos para optar al título de Doctor en Ciencias Administrativas.

De LaTorre, L., \& Afan, K. (2017). CULTURA ORGANIZACIONAL Y LARELACIÓNCON EL DESEMPEÑO LABORAL EN LOS TRABAJADORES DE LA OFICINA DE DESARROLLO TÉCNICO DE LA BIBLIOTECA NACIONAL DEL PERU. Lima, Perú:

Trabajo especial de grado de la Universidad San Ignacio de Loyola.

Diehl, S., Schaefer, S., \& Terlutter, R. (2019). Is my Companyreally doing good? Factors influencing employee's evaluation of the authenticity of their company's corporate social responsibility engagement. Journal of business research , 101-143.

Espinoza, A., \& Gaspar, J. (2018). Cultura Organizacional y Bienestar Laboral en profesionales de salud del centro de salud Ascención Huancavelica.Universidad Nacional de Huancavelica. Perú. Huancavelica-Perú: Trabajo especial de grado de la Universidad Nacional de Huancavelica para optar al título de Licenciada en Enfermeria.

Flebes, J., \& Oreja, J. (2018). Factores Externos e Internos determinantes de la Orientación de la Cultura Estratégica de la Empresas. Investigaciones Europeas de Dirección y Economía de la empresa 14(1) ,13-32.

Granados, L. (2017). Desarrollo de una cultura empresarial contra el paradignma de las agencias publicitarias. Biblioteca Digital de la Universidad de Sonora. Recuperado el 28 de Junio de 2021, de http://www.bidi.uson.mx/TesisIndice.aspx?tesis=19652 


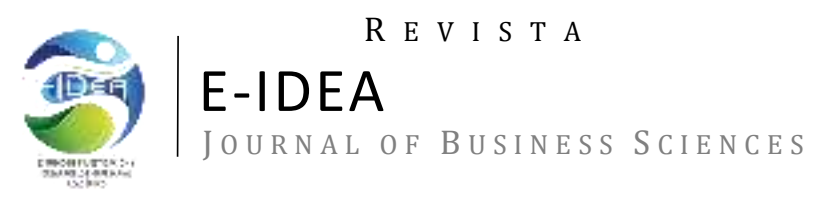

Jiménez, J. (2016). La cultura organizacional y su relación con el desempeño laboral del personal de la empresa HumanaS.A Pontificia Universidad Católica del Ecuador. Quito, Ecuador: Trabajo especial de grado de la Pontificia Universidad Católica del Ecuador para optar al título de Ingenieria Comercial.

Lee, K., Lee, S., \& Youn, H. (2018). Effects of corporate social responsibility on employees in the casino industry. Tourism Management, 328- 335.

Loufatt, E. (2012). Administración del Potencial Humano (Vol. 1). (2da, Ed.) Buenos Aires, Argentina: CENGAGE.

Mendez, C. (2019). Elementos para la relación entre cultura organizacional y estrategia. Universidad \& Empresa Universidad del Rosario, 21 (31), 136 -169.

Palella Stracuzzi, S., \& Martins Pestana, F. (2010). Metodologia de la Investigación Cuantitativa. Carcas, Venezuela: FEDUPEL, Fondo Editorial de la Universidad Pedagógica Experimental Libertador.

Raffino, E. (2020). Cultura organizacional. Recuperado el 28 de Junio de 2021, de https://concepto.de/cultura-organizacional/

Robbins, S. (2004). Comportamiento Organizacional (10 a ed.). México: Pearson Educacion. Robbins, S., \& Coulter, M. (2014). Administración (12a ed ed.). Ciudad de México: Pearson.

Sira, H. (2020). Clima organizacional y su relación con el desempeño laboral docente en la Seccion de Asignaturas Generales UNEXPO. Congreso Internacional de Metodologia de la Investigación para ciencias tecnologia e innovación. Recuperado el Junio de 2021, de https://www.youtube.com/watch?v=p5ajuE17AS8 
Su, L., \& Swanson, S. (2019). Impacto percibido de la responsabilidad social corporativa en el bienestar y los comportamientos ecológicos de apoyo de los empleados del hotel: el papel mediador de la relación empleado-empresa. Tourism management 72 , 437450 .

Toro F. (2010). Validación psicométrica de un conjunto de instrumentos que evalúan efectos individuales de diversos factores psicosociales. Revista Interamericana de Psicología Ocupacional , 29 (2), 193- 225.

Toro, F. (2002). Desempeño y Productividad. Medellín: Cincel. 